\section{TUMOR CELL-INTRINSIC MTORC1 SIGNALING THROUGH RAPTOR MAKES MELANOMA AND OVARIAN CANCER IMMUNOTHERAPY RESISTANT BY REGULATING INTERFERON-GAMMA RESPONSIVENESS AND PROMOTING TUMOR-INITIATING CELLS}

${ }^{1}$ Harshita Gupta* ${ }^{2}$ Suresh Kari, ${ }^{2}$ Emily Salinas, ${ }^{2}$ Haiyan Bai, ${ }^{2}$ Erica Osta, ${ }^{2}$ Anand Kornepati, ${ }^{2} J$ uan Wang, ${ }^{2}$ Xinyue Zhang, ${ }^{2}$ Yidong Chen, ${ }^{2}$ Ratna Vadlamudi, ${ }^{2}$ Tyler Curiel. ${ }^{1}$ UTHSCSA, San Antonio, TX, USA; ${ }^{2}$ UTHSA, San Antonio, TX, USA

Background Although immunotherapy can induce durable antitumor response in multiple cancers, immune checkpoint blockade (ICB) therapy resistance in ovarian cancer and melanoma remains problematic. Here, we report that tumor cell-intrinsic mTORC1 regulates ICB response through mTORC1 defining subunit Raptor (Rptor) by modulating interferon-gamma (IFNg) resistance and tumor-initiating cell (TIC) virulence.

Methods We knocked down two distinct mTORC1 signaling components: Rptor (Rptorlo, aids in mTORC1 assembly) and Lamtor1 (Ltor1lo, docks mTORC1 on lysosomes) in murine ovarian cancer ID8agg and melanoma B16 cells. PD-L1 was CRISPR knocked out in B16 and human ovarian cancer line ES2. Mice with tumors were treated with a-PD-L1 \pm a-CD8 antibody. TICs were estimated by flow-cytometry. ${ }^{1}$

Results Rptorlo B16 and ID8agg, but not Ltor1lo B16 tumors grew slower and were a-PD-L1 responsive unlike control (ctrl) in WT mice. We noted that ctrl and Rptorlo B16 and ID8agg cells expressed similar surface PD-L1 in vitro. Thus, Rptor suppresses a-PD-L1 response in ICB-resistant tumors. Tumor immune analysis revealed increased $\mathrm{CD} 8+\mathrm{T}$ cell $\%$ and a trend to increased IFNg $+\mathrm{CD} 8+\mathrm{T}$ cells in a-PD-L1 treated Rptorlo, but not ctrl B16. Rptorlo a-PD-L1 efficacy was lost with a-CD8 and in IFNg knockout mice. In vitro, IFNg suppressed Rptorlo ID8agg proliferation, unlike ctrl. These data suggested that lack of Rptor makes tumors ICB responsive, possibly by making tumors IFNg-sensitive and increasing IFNg $+\mathrm{CD} 8+\mathrm{T}$ cells. Further, tumor and draining lymph node (DLN) TCF1+PD-1+ T cell stem cells (critical for aPD-L1/ PD-1 success ${ }^{2}{ }^{3}$ ) were significantly higher in a-PD-L1 treated Rptorlo tumors. Thus, tumor Rptor status could regulate tumor microenvironment and distal DLN immune landscape on a-PD-L1 treatment.We previously published that mTORC1 promotes PD-L1-dependent tumor proliferation, TIC virulence $^{1} 4$ PD-L1KO B16 and ES2 cells expressed similar total Rptor protein. However, lower levels of Rptor were loaded in mTOR complex in absence of PD-L1, as assessed by a-mTOR immunoprecipitation, suggesting that pro-tumorigenic Rptor functions were downstream of, and dependent on PD-L1. Successful Rptorlo aPD-L1 treatment reduced TIC in vivo, an effect reversed in absence of $\mathrm{CD} 8+\mathrm{T}$ cells or host IFNg. Inhibiting ID8agg mTORC1 with rapamycin reduced stemness genes oct4, nanog expression by QPCR. Further, ID8agg Rptorlo TIC formed significantly smaller tumors versus ctrl TIC in immune-compromised NSG mice, confirming their reduced virulence. Rptor, but not Ltor1, expression inversely correlated with tumor CD8+ infiltrate in IMvigor210 trial, and strongly with TIC gene signature in ovarian cancer patients. $^{5} 6$

Conclusions Tumor-cell intrinsic Rptor modulates ICB resistance, IFNg responsiveness, immune microenvironment, and TIC virulence.

Acknowledgements N/A

Trial Registration N/A

\section{REFERENCES}

1. Gupta HB, et al. Tumor cell-intrinsic PD-L1 promotes tumor-initiating cell generation and functions in melanoma and ovarian cancer. Signal Transduct Target Ther 2016;1,Article number:16030.

2. Im SJ, et al. Defining CD8+ T cells that provide the proliferative burst after PD-1 therapy. Nature 2016;537:417-421.

3. Dammeijer $F$, et al. The PD-1/PD-L1-checkpoint restrains T cell immunity in tumordraining lymph nodes. Cancer Cell 2020;38:685-700.

4. Clark CA, et al. Tumor-intrinsic PD-L1 signals regulate cell growth, pathogenesis, and autophagy in ovarian cancer and melanoma. Cancer Res 2016;76:69646974.

5. Smith BA, et al. A human adult stem cell signature marks aggressive variants across epithelial cancers. Cell Rep 2018;24:3353-3366.

6. Hoffman-Censits $\mathbf{J H}$, et al. IMvigor 210, a phase II trial of atezolizumab (MPDL3280A) in platinum-treated locally advanced or metastatic urothelial carcinoma (mUC). J Clin Oncol 2016;34, no.2_suppl:355-355.

http://dx.doi.org/10.1136/jitc-2021-SITC2021.298 